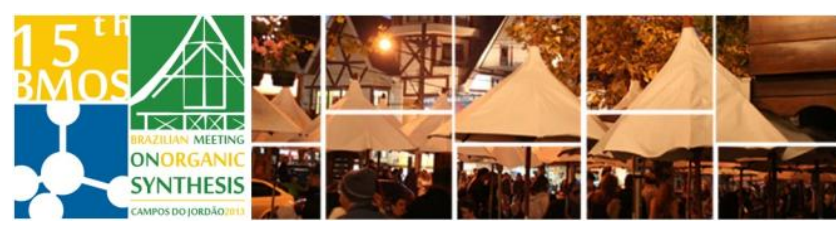

\title{
Rational Synthesis, Photophysical and Biochemical Evaluation of Fluorescents Chalcones as Inhibitors of Cruzain from Trypanosoma cruzi
}

\author{
Wender A. Silva ${ }^{\text {a }}$, Lennine R. Melo ${ }^{a}$, Guilherme R. Oliveira, ${ }^{\text {b }}$ Camilla C. C. Silva, ${ }^{\text {c }}$ \\ Gustavo H. G. Trossinic \\ a) Institute of Chemistry, IQ-UnB, University of Brasilia, 70904-970, P.O.Box 4478, Brasília, Brazil. b)
} Institute of Chemistry, University Federal of Goiás, C.P. 131, CEP 74001-970, Goiânia, Brazil C) Phaculty of Pharmaceutical Sciences, FCF-USP, University of São Paulo, 05508-900, São Paulo, Brazil

${ }^{*} e$-mail corresponding author: lennine@unb.br

Keywords: Fluorescents Chalcones, Docking, Cruzain

\section{INTRODUCTION}

Chagas' disease is an infection caused by the protozoan parasite Trypanosoma cruzi, considered by World Health Organization as a neglected disease it causes thousands of deaths each year. Only drugs, nifurtimox and benznidazole, are using against this disease, however it are effective just in the acute phase and are highly toxic. Thus, new drugs are urgently needed. ${ }^{1}$ Cruzain, a cysteine protease, has been selected as an attractive target for the development of new antitrypanosomal agents. $^{2}$ In this context, chalcones (1,3-diaryl-2propen-1-ones) belong to the flavonoid family, and commonly possess interesting biological activities, as reviewed elsewhere, have presented a promising inhibitory activity against cruzain. ${ }^{3,4}$

\section{RESULTS AND DISCUSSION}

The thirteen chalcones were synthesized based on designed fluorescents by a Claisen-Schmidt condensation as shown scheme 1 .

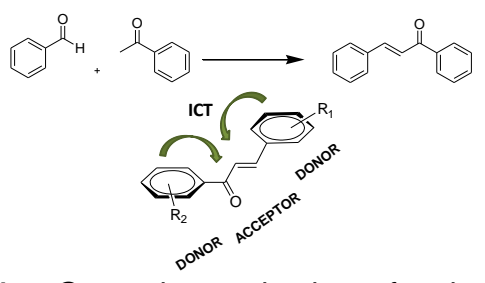

Scheme 1. General synthesis of chalcone and rationalization of the designed fluorescent chalcone derivative.

The photophysical properties of the novel compound was evaluated by spectrophotometric and spectrofluorimetric analyses. Initially, to investigate the ICT possibility of these new compounds, solvatochromic effects (by spectrophotometric and spectrofluorometric analyses) were carried out. The best quantum yields of chalcones are presenting groups in meta and para positions as showed on the chart 1.
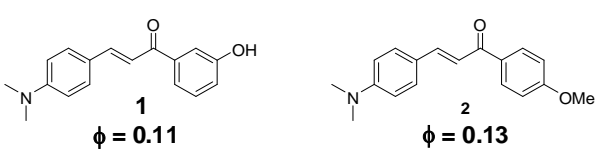

Chart 1. The best quantum yield for the highlighted chalcones.

Subsequently structural analyzes were performed the structural complementarity between chalcones, by best ranked docking poses, have suggested aromatic groups in the S2 sub-pocket of cruzain, were observed for all compounds, with the exception of one. These results corroborated with literature and the frame conducive to attack the Cys25 cruzain, inhibition mechanism proposed for this chemical class, (Figure 1).

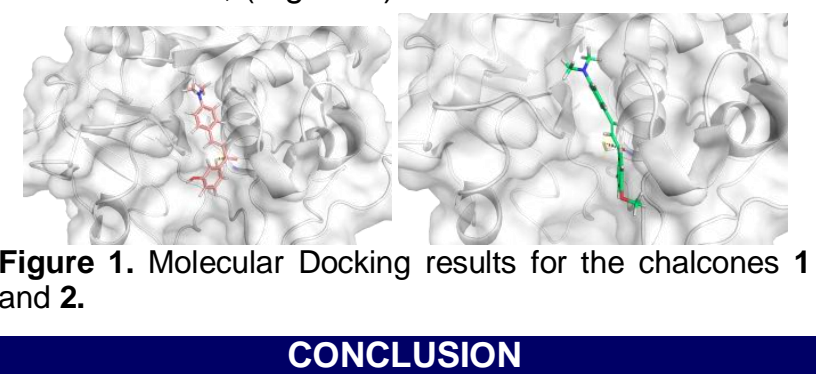

The compounds evaluated were promising in silico. Furthermore, the compounds will be tested by biochemistry inhibitory assays in cruzain to direct the synthesis of a library of antichagasic candidates.

\section{ACKNOWLEDGEMENTS}

CNPq, DPP-UnB FAPESP

\section{REFERENCES}

${ }^{1}$ Z. Nowakowska, Eur. J. Med. Chem., 2007, 42, 125.

${ }^{2}$ Sajid, M.; McKerrow, J. H. Mol. Biochem Parasitol. 2002, 1221, 159.

${ }^{3}$.M.; Xavier, M. E.; Soares, T. A.; Gomes, A. F.; Gozzo, F. C.; Silva, W. A.; Neto, B. A. D. Org. Biomol. Chem., 2013, 11, 4764.

${ }^{4}$ Borchhardt, D. M.; Mascarello, A.; Chiaradia, L. D.; Nunes, R. J.; Oliva, G.; Yunes, R. A.; Andricopulo, A. D. J. Braz. Chem. Soc. 2010, 21,142. 